\title{
Atomic-Resolution Imaging of Crystals Using Charge Flipping and Precession Electron Diffraction
}

\author{
Hefei Hu, ${ }^{1,3}$ and J. M. Zuo ${ }^{2,3}$
}

${ }^{1}$ Department of Physics, University of Illinois at Urbana-Champaign, 1110 W. Green St., Urbana, IL, 61801

2 Department of Materials Science and Engineering, University of Illinois at Urbana-Champaign, 1304 W. Green St. Urbana, IL, 61801

${ }^{3}$ F. Seitz Materials Research Lab, University of Illinois at Urbana-Champaign, 104 S. Goodwin Ave., Urbana, IL, 61801

Solving the structure of crystals at atomic resolution is essential to further understand the properties of materials. While the resolution of electron direct imaging is limited by lens aberrations, electron diffraction resolution, however, is only limited by atomic scattering. Electron diffraction also can work with radiation sensitive materials. Retrieving the missing phases of electron diffraction patterns is required to reconstruct the image. A simple, but surprisingly effective, phase retrieval algorithm, called charge flipping (CF), was proposed in 2004 [1]. The charge densities in case of X-ray diffraction are sign-reversed when they are below a threshold in an iterative cycle of Fourier transforms. No support constraint is needed in the CF algorithm, and it does not require the knowledge of atom types or symmetry. The challenge of applying $\mathrm{CF}$ algorithm on electron diffraction patterns rises from multiple scattering, which causes the diffracted intensities to deviate far from the so-called kinematical intensities obtained for very thin samples. This discrepancy can be remedied by the precession technique [2]. Commercial precession units are now available for existing electron microscopes. Here, we report an optimization of the CF algorithm for reconstructing atomic-resolution imaging using precession electron diffraction (PED) data based on simulations of silicon crystals along the [110] zone axis. Applications to experimental data will be presented in the talk.

The PED patterns used here are simulated using the Bloch wave method by tilting the incident electron beam one angle at a time and average over a large number of angles around the precession circle. The PED patterns for four different thicknesses, $250 \AA, 500 \AA, 1000 \AA$ and $2000 \AA$, are calculated, and intensities are extracted from the patterns by using the program of QED [3]. Comparisons of PED diffraction intensities and theoretical structure factors are shown in Fig.1. Both the PED amplitudes, F(PED), and theoretical structure factors, F(kin), are normalized to the strongest amplitude respectively. The approximate linear dependence of F(PED) on F(kin) suggests that kinematical interpretation on PED datasets is reasonable for small thicknesses, especially for the medium and strong reflections. As the specimen thickness increases, deviations from the linear dependence increase. Deviation can be very large for very weak (kinematical forbidden) reflections. To minimize the multiple scattering effects, we modified the $\mathrm{CF}$ algorithm by intentionally reducing the contribution of weak beam amplitudes. Fig.2 demonstrates the projected density maps reconstructed from the datasets in Fig.1. The dumbbell structure of the Si crystal in [110] direction is clearly resolved even for $2000 \AA$ thickness. This preliminary work on atomic-resolution imaging of Si crystals shows that the combination of the CF algorithm and the PED technique can be extended to crystals of reasonable thicknesses. 
References

[1] G. Oszlányi and A. Sütő, Acta Cryst. (2004), A60, 134-141

[2] R. Vincent and P. Midgley, Ultramicroscopy, (1994), vol.53, issue 3, 271-282

[3] Http://www.emlabsoftware.com
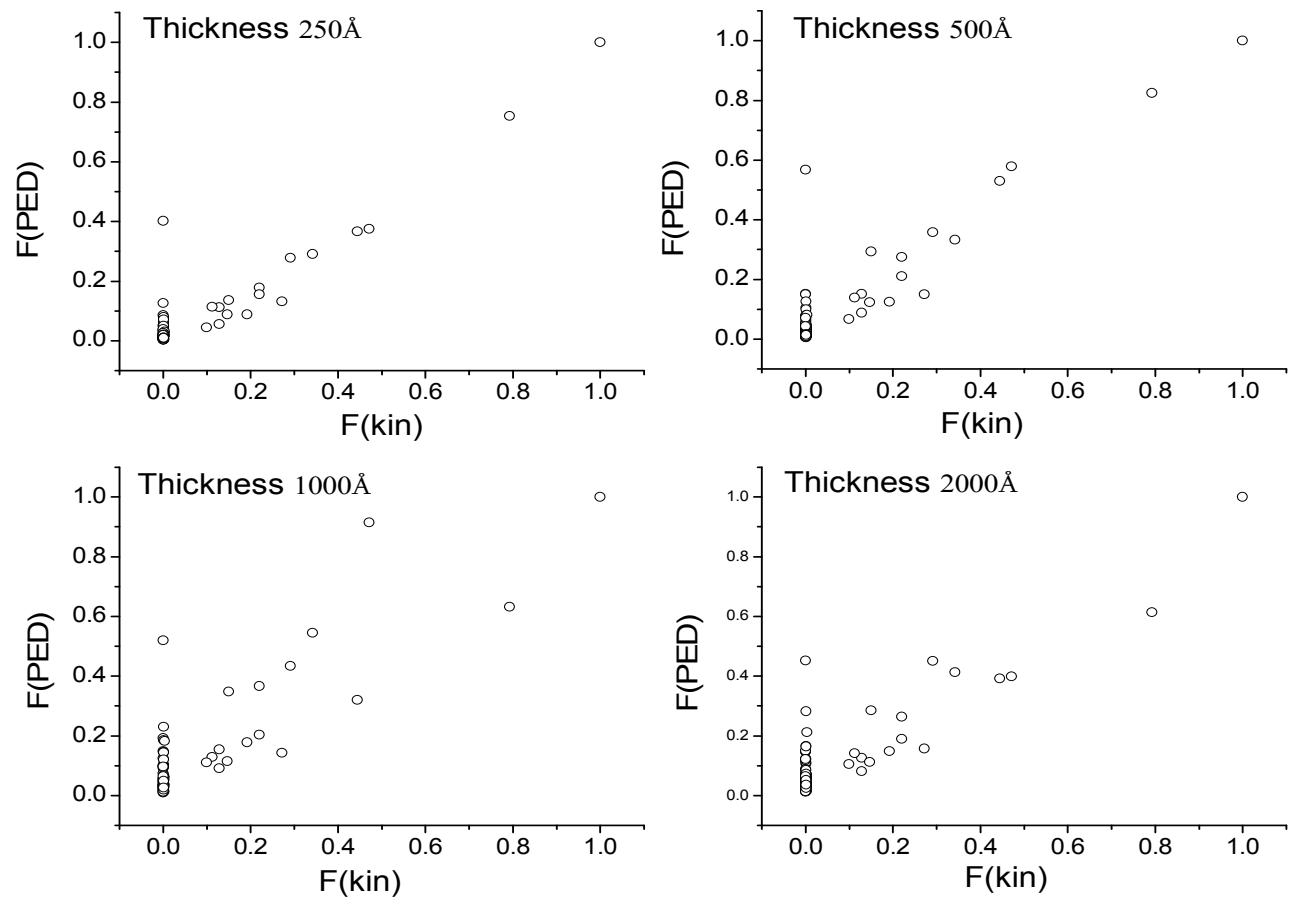

Fig.1 Comparison between simulated precession electron amplitudes on Si (110) diffraction patterns and theoretical structure factors for different thicknesses. PED amplitudes, F(PED), are the square root of the measured intensities.
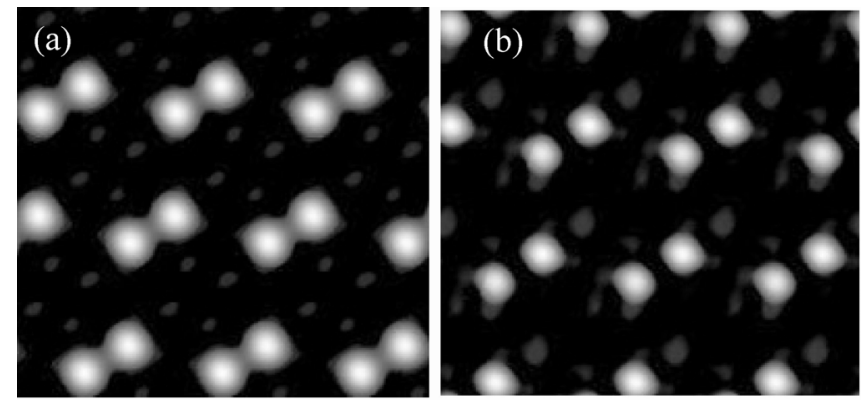

Fig.2 Projected density maps, reconstructed from the simulated PED amplitudes, along the Si [110] zone axis for thickness of (a) $250 \AA$, (b) $500 \AA$, (c) $1000 \AA$ and (d) $2000 \AA$.
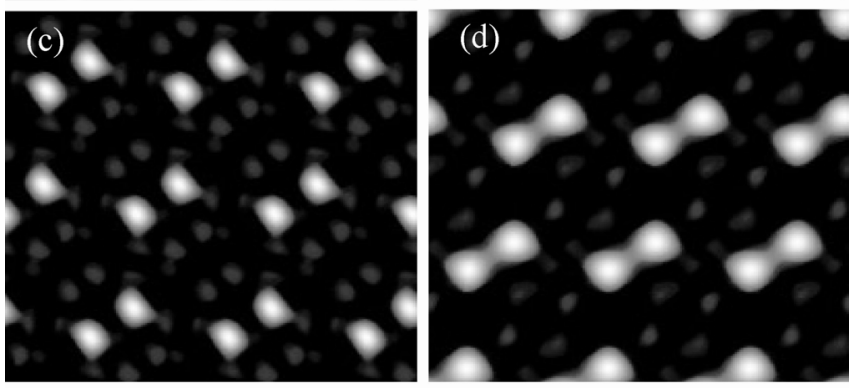